\title{
Population dynamics of the potato cyst nematode, Globodera pallida in relation to temperature, potato cultivar and nematicide application
}

\author{
by Kaczmarek, A.M., Back, M. and Block, V.C.
}

Copyright, Publisher and Additional Information: This is the author accepted manuscript. The final published version (version of record) is available online via Wiley. This article may be used for non-commercial purposes in accordance with Wiley Terms and Conditions for Self-Archiving.

Please refer to any applicable terms of use of the publisher.

DOI: https://doi.org/10.1111/ppa.13002

Kaczmarek, Back, M. and Block, V.C. 2019. Population dynamics of the potato cyst nematode, Globodera pallida in relation to temperature, potato cultivar and nematicide application. Plant Pathologyl. 


\section{Population dynamics of the potato cyst nematode, Globodera}

\section{2 pallida, in relation to temperature, potato cultivar and nematicide}

\section{3 application}

4 A. M. Kaczmareka , M. Back ${ }^{b}$ and V. C. Blok ${ }^{a *}$

5 a James Hutton Institute, Invergowrie, Dundee, DD2 5DA; and ${ }^{\mathrm{b} H a r p e r}$ Adams University,

6 Harper Adams, TF10 8NB, UK

$7 \quad$ *E-mail: Vivian.Blok@hutton.ac.uk

9 The impact of increasing temperatures on the population dynamics of the soil-dwelling

10 nematode Globodera pallida, a persistent and economically important pest of potatoes, was

11 investigated. The reproductive factor (final populationinitial population) and length of life cycle were found to be temperature sensitive. Pot experiments performed over 4 months allowed comparison of the effect on development of G. pallida of two temperature regimes: an average temperature comparable to current field conditions $\left(14.3^{\circ} \mathrm{C}\right)$ and an average temperature above current field conditions $\left(17.3^{\circ} \mathrm{C}\right)$. A larger second generation of juveniles was observed at $17.3^{\circ} \mathrm{C}$ compared to $14.3^{\circ} \mathrm{C}$. Multiplication of G. pallida at field sites in

17 Shropshire and East Lothian (average soil temperatures of 14.1 and $15.1{ }^{\circ} \mathrm{C}$, respectively, during potato cropping) was also examined. A quantitative PCR assay and visual examination of roots were used to monitor the dynamics of the G. pallida populations in both field sites at 4-weekly intervals. Four cultivars, Desirée, Cara, Maris Piper and Estima, were grown with and without nematicide treatments. Nematicide treatments suppressed population increases at both sites. Females were observed on the roots of cvs Cara and Desirée at the end of the 

generation. 2000).

Keywords: population dynamics, potato cyst nematode, second generation, temperature, nematicide treatment

\section{Introduction}

The potato cyst nematodes (PCN) Globodera rostochiensis and Globodera pallida are major parasites of potatoes and other members of the Solanaceae family. Infected plants typically have a smaller root system (Trudgill \& Cotes, 1983), have a diminished capacity to take up nutrients from the soil and are adversely affected by water stress or disturbance of nutrient metabolism. According to the EPPO Global Database (2019), G. rostochiensis and G. pallida have been detected in 72 and 49 countries respectively. In the UK, the direct and indirect crop losses caused by PCN were valued at $9 \%$ of yield annually (Evans, 1993) and the economic cost of PCN to the UK processing and fresh market potato industry was estimated at $c$. $£ 26$ million in 2009 (Twining et al., 2009). Increasing management costs after implementation of the PCN Directive (2007/33/EU), which requires improved pre-planting soil testing for all seed crops and an annual survey of $0.5 \%$ of ware land, also add to the economic impact of PCN. In fields where PCN are detected, the directive prohibits the growing of seed potatoes, and ware potatoes may only be grown under an officially approved control programme that includes using resistant varieties, nematicides or other control measures (Hockland et al.,

The population dynamics of G. pallida and G. rostochiensis and associated yield loss of the potato crop differ greatly among years and locations (Greco et al., 1982; Seinhorst, 
1982). As mentioned above, damage caused by PCN, particularly G. rostochiensis, can be

47 minimized by the selection of resistant potato cultivars within a rotation cycle (Trudgill,

48

49

50

51

52

53

54

55

56

57

58

59

60

61

62

63

64

65

66

67

68

69
1986). However, with susceptible cultivars, factors including the amount of the initial

population of PCN (Trudgill et al., 2014), environmental factors (Jones, 1983) and soil type

(Trudgill, 1986; Elston et al., 1991) can affect multiplication rates. In general, the larger the population of Globodera spp. in the field at planting, the greater the yield losses (Trudgill et al., 2014; Seinhorst, 1982), although it is also known that PCN reproduction is density dependent (Trudgill et al., 2014) and at lower densities the multiplication rate is higher.

In the last century, global temperatures have been increasing due to climate change and the effect of this trend on crop losses due to plant pests and diseases, including soil dwelling organisms such as PCN, is of concern (Ellenby \& Smith, 1975; Stanton \& Sartori, 1990; Munir et al., 2009, Ebrahimi et al., 2014; Jones et al., 2017; Skelsey et al., 2018).

Recently Jones et al. (2017) and Skelsey et al. (2018) have reported on the potential impact of climate change in the UK on the potato cyst nematodes G. rostochiensis and G. pallida based on experiments conducted in controlled environments. While the reports differ in some respects, they generally agree that increases in soil temperatures from current levels are likely to lead to increased multiplication of PCN, particularly G. rostochiensis. Globodera pallida is more adapted to cooler temperatures than G. rostochiensis and, hence, increases in soil temperatures above current levels are more likely to benefit the latter species. However, the relationship between temperature and population dynamics for G. pallida has not been examined under different in vivo field conditions in the UK. The aim of the present study was to investigate the multiplication and development of PCN at two field sites in the UK and compare this with multiplication and development in controlled environments. 


\section{Nematodes and plant material}

Globodera pallida population (Lindley) (Pa2/3) cysts from The James Hutton Institute (JHI)

73

74

75

PCN collection were used for experiments in temperature-controlled conditions. This

population had been maintained in glasshouse conditions $\left(20^{\circ} \mathrm{C} 16 \mathrm{~h}, 16{ }^{\circ} \mathrm{C}, 8 \mathrm{~h}\right)$ for over 30 years at the JHI. Cysts were collected on a $250 \mu \mathrm{m}$ sieve, randomly selected and packed into nylon bags containing 30 cysts per bag. Cysts of G. pallida (Lindley) were also used for the validation of qPCR assays.

The susceptible cultivar Desirée and the moderately resistant cultivar Morag, which has resistance to G. pallida derived from Solanum vernei (Phillips \& Trudgill, 1998), were used in a pot experiment to examine the life cycle of G. pallida in temperature-controlled conditions. For the field experiment, four susceptible cultivars were used at each site (Desirée, Maris Piper, Cara and Estima) whilst the susceptible cultivar Edzell Blue, which produces blue tubers, was used to produce guard rows as an additional measure to distinguish the boundaries of the plots. The four cultivars selected are commonly grown in the UK and are classified as early (Estima), main crop (Desirée and Maris Piper) and late cropping (Cara).

Occurrence of juveniles, males and cysts in the soil under different temperature regimes in controlled environments

Growth cabinets were used to monitor the occurrence of hatched juveniles, free-living adult males and new cysts of G. pallida in the soil at two different temperature regimes. The growth cabinets (Phytotron model 1700; Sanyo) were set as follows: (i) $16{ }^{\circ} \mathrm{C}$ for $16 \mathrm{~h}$ with 
light $\left(525 \mu \mathrm{mol} \mathrm{m} \mathrm{m}^{-2} \mathrm{~s}^{-1}\right)$ and $11^{\circ} \mathrm{C}$ for $8 \mathrm{~h}$ with no light and (ii) $19{ }^{\circ} \mathrm{C}$ for $16 \mathrm{~h}$ with light and $14{ }^{\circ} \mathrm{C}$ for $8 \mathrm{~h}$ with no light. Soil temperatures were recorded every $3 \mathrm{~h}$ in the pots in the growth cabinet experiment with DS1920-F5 Temperature ibuttons (HomeChip Ltd) placed into the soil at a depth of $10 \mathrm{~cm}$. The average soil temperatures of these regimes were 14.3 and $17.3{ }^{\circ} \mathrm{C}$, respectively. The relative humidity inside the growth cabinets was maintained at $75 \%$. The accumulated day degrees (DD) were calculated using the formula $\mathrm{DD}=\left(\mathrm{T}_{\text {mean }}-\right.$ $\left.\mathrm{T}_{\text {base }}\right) \times$ Days, where $\mathrm{T}_{\text {mean }}$ is the mean temperature of that day and $\mathrm{T}_{\text {base }}$ is the base temperature needed for G. pallida to hatch, which was $4{ }^{\circ} \mathrm{C}$ according to Ebrahimi et al. (2014).

Tuber pieces ( $3 \mathrm{~cm}$ diameter) with a single sprout were cut with a melon scoop from tubers of cvs Desirée or Morag and planted in pots containing $500 \mathrm{~g}$ autoclaved sand:loam (50:50). Plants (96 per cultivar) were grown in the greenhouse until they were approximately $10 \mathrm{~cm}$ high before being moved to the growth cabinets. The plants were arranged in a randomized design in two growth cabinets for each temperature regime and, 2 days later, a nylon cyst bag containing 30 cysts was planted into the soil of each pot, at a depth of about 5 $\mathrm{cm}$, beside the tuber piece. Five weeks after inoculation, the cyst bags were removed from the pots to distinguish between juvenile nematodes originating from these cysts and those from new females. The bags were removed with tweezers by pinching the top of the bag at the soil surface and pulling from the soil.

Three pots per temperature and cultivar were randomly selected at 7-day intervals for 16 weeks. The juveniles and males were extracted from the soil using the Baermann funnel method (Viglierchio \& Schmitt, 1983). After 2 days, nematodes were collected and the numbers of juveniles and males were counted with a microscope. Additionally, the total number of cysts per pot was determined in weeks 8 to 16 by using acetone flotation to 
recover the cysts that were collected in the sieves used with each Baermann funnel (Brodie $e t$ $a l,, 1976)$.

$120 \quad$ Field trials

121 In 2011, field trials took place in two locations that were naturally infested by G. pallida:

122 East Lothian (lat 56.0170, long -2.8375) and a site near Harper Adams University, Newport 123 (lat 52.7797, long -2.4275). The soil temperatures were monitored with DS1920-F5 temperature monitoring ibuttons that were placed in the centre of two neighbouring potato ridges at a depth of $20 \mathrm{~cm}$. Temperatures were recorded every $3 \mathrm{~h}$ during the trials. time of planting potatoes in East Lothian and fosthiazate $\left(30 \mathrm{~kg} \mathrm{ha}^{-1}\right)$ was applied immediately before planting at the site near Harper Adams. The farm managers were responsible for all agricultural operations, including nematicide application, fertilization and application of other crop protection products at each site. four cultivars (Desirée, Maris Piper, Cara and Estima) in a randomized design. One replicate consisted of three tubers of each cultivar, planted $25 \mathrm{~cm}$ apart. Between trials and at the end of each row, two tubers of the guard plants (Edzell Blue) were planted $25 \mathrm{~cm}$ from the experimental blocks and from each other. The experiments were set up on 4 April 2011 and 4 May 2011 at the Harper Adams and East Lothian sites, respectively. experimental block with a $15 \mathrm{~mL}$ corer. Soil samples were subsequently collected at 
140 October in East Lothian. During harvesting, approximately $5 \mathrm{~L}$ of soil, containing and

141 surrounding the roots of each of the three plants per replicate, was collected with a spade,

142 combined and mixed in a bucket. Subsequently, a subsample (at least $500 \mathrm{~g}$ ) was placed in a

143 labelled bag. Soil samples were air dried in the glasshouse by spreading each in a plastic tray

$144(38 \times 24 \times 5 \mathrm{~cm})$ and, when dry, a $400 \mathrm{~g}$ soil sample was packaged in a labelled paper bag

145 and transferred to Science and Advice for Scottish Agriculture (SASA, UK) for cyst

146 extraction using their automated soil washing carousel (Meku). The wet filter papers on

147 which the cysts and debris were collected following their extraction from the soil with the

148 carousel, were dried and the cysts were further purified by acetone flotation (Brodie et al.,

149 1976) and then transferred into a $2 \mathrm{~mL}$ Eppendorf tube384

150

151

To monitor nematode development, root samples of cvs Desirée and Cara were

152 collected when each trial was harvested. The root systems for each replicate of three plants

153 were cut off and the remaining soil was gently shaken off. Roots were chopped into 5-7 cm

154 segments and stored in FAA (formalin-acetic-alcohol, 2:1:10) solution (Hooper, 1970).

155 Later, a $0.5 \pm 0.2 \mathrm{~g}$ subsample was stained with acid fuchsin (Bridge et al., 1981) and then 156 examined by microscope (Olympus S7-ST).

158 DNA extraction from soil samples

159 The floats obtained following acetone purification were placed in $2 \mathrm{~mL}$ Eppendorf tubes with

160 two metal beads per tube and pulverized in a mixer mill MM300 (Retsch) for 1.5 min at 30

$161 \mathrm{~Hz}$. The resulting powder was mixed with $0.5 \mathrm{~mL}$ GeneScan lysis buffer (Neogen Europe

$162 \mathrm{Ltd})$ and ground again for $30 \mathrm{~s}$. Samples were centrifuged for $15 \mathrm{~s}$ at $15600 \mathrm{~g}$ and then $5 \mu \mathrm{L}$ 
163

164

165

166

167

of $20 \mathrm{mg} \mathrm{mL}^{-1}$ proteinase $\mathrm{K}$ in $40 \%$ (v/v) glycerol (Sigma-Aldrich) was added and incubated for $1 \mathrm{~h}$ at $65{ }^{\circ} \mathrm{C}$. After incubation, $0.5 \mathrm{~mL}$ chloroform:isoamyl alcohol (24:1) (Sigma-

Aldrich) was added and mixed by inverting the tube five times. Samples were centrifuged for $10 \mathrm{~min}$ at $15600 \mathrm{~g}$ in an Eppendorf centrifuge, the upper aqueous phase $(450 \mu \mathrm{L})$ was transferred into a new tube and $360 \mu \mathrm{L}$ of ice-cold propan-2-ol (VWR) was added and mixed thoroughly. Subsequently, samples were incubated for $30 \mathrm{~min}$ at $20{ }^{\circ} \mathrm{C}$, centrifuged for 10 min at $15600 \mathrm{~g}$ and the pellet was retained. The pellet was washed twice with $0.5 \mathrm{~mL} 75 \%$ ethanol and centrifugation for $5 \mathrm{~min}$ at $15600 \mathrm{~g}$ and then resuspended in $100 \mu \mathrm{L}$ sterile $\mathrm{dH}_{2} \mathrm{O}$.

The resulting DNA was further purified on PVPP columns. First, a $600 \mu \mathrm{L}$ suspension of $10 \%$ PVPP (Sigma-Aldrich) was made up with sterile distilled water and transferred to a spin column (NBS Biologicals) in a $2 \mathrm{~mL}$ microcentrifuge tube. Tubes were centrifuged at $11000 \mathrm{~g}$ for $1 \mathrm{~min}$, the catch-tube was emptied and the centrifugation was repeated (the spin column was rotated $180^{\circ}$ within the centrifuge before the second spin). The resuspended DNA was transferred to the spin column and centrifuged at $11000 \mathrm{~g}$ for $1 \mathrm{~min}$. Purified eluate was transferred to a new sterile $1.5 \mathrm{~mL}$ Eppendorf tube and stored at $-20{ }^{\circ} \mathrm{C}$.

\section{PCN quantification by qPCR}

The oligonucleotide design and optimization of specificity and sensitivity of the PCN qPCR assay was previously described by Reid et al. (2010). The primers and probes used were designed for the rDNA ITS1 region of G. pallida and G. rostochiensis; primer 1 (forward) 5'CGTTTGTTGTTGACGGACAYA-3', primer 2 (reverse) 5'-

GGCGCTGTCCRTACATTGTTG-3', G. pallida MGB probe 5'-6FAMCCGCTATGTTTGGGC-3', G. rostochiensis MGB probe 5'-6FAM- 
CCGCTGTGTATKGGC-3'. All DNA from the field samples was tested for both PCN species; however, no G. rostochiensis was detected when compared to the DNA standard prepared from G. rostochiensis cysts from the JHI collection. To determine whether the assay was valid over a wide range of cyst densities, DNA was extracted from G. pallida (Lindley) cysts that had been recovered from a 1:1 sand:loam mixture (260 g per pot) with 12 to 644 cysts per pot (average number of eggs $205.4 \pm 20.3$ per cyst). DNA was extracted from these cysts as described for the field samples.

Real-time qPCR reactions were set up using a Genesis Workstation 150 (Tecan Inc.) in 96-well plates (Applied Biosystems/Thermo Fisher Scientific) at SASA, UK. The $30 \mu \mathrm{L}$ reactions contained $15 \mu \mathrm{L}$ Environmental BLUE Ready Mix (Eurogentec Ltd), $1.25 \mu \mathrm{L}$ each of the forward and reverse primers for either species of PCN $\left(5 \mathrm{pmol} \mu \mathrm{L}^{-1}\right), 1.25 \mu \mathrm{L}$ of either the G. pallida- or G. rostochiensis-specific probe $\left(5 \mathrm{pmol} \mu \mathrm{L}^{-1}\right), 6.25 \mu \mathrm{L}$ distilled water (Sigma-Aldrich) and either $5 \mu \mathrm{L}$ DNA that had been extracted from samples and diluted 1:10 with $\mathrm{H}_{2} \mathrm{O}$, or $5 \mu \mathrm{L} \mathrm{H}_{2} \mathrm{O}$ as a negative control. The reactions were then aliquoted in triplicate into 384-well plates (Applied Biosystems) using a Genesis robot (Tecan). Standards (in triplicate) were created from G. pallida cyst DNA dilutions of 10, 1, 0.1, 0.01 and $0.001 \mathrm{ng}$ $\mu \mathrm{L}^{-1}$. Amplification was performed in an ABI 7900HT (Applied Biosystems) real-time machine run in the standard mode with the following cycling conditions: $50{ }^{\circ} \mathrm{C}$ for $2 \mathrm{~min}, 95$ ${ }^{\circ} \mathrm{C}$ for $5 \mathrm{~min}$, followed by 40 cycles of $95^{\circ} \mathrm{C}$ for $15 \mathrm{~s}$ and $60^{\circ} \mathrm{C}$ for $1 \mathrm{~min}$. Linear regression of the quantity of copies (qty values) versus egg number and calculation of the corresponding $R^{2}$ value were performed using EXCEL v. 16.0.4456.1003 (Microsoft) and GENSTAT v. 16.1.010916.

The average value of qty values obtained from the qPCR performed on DNA extracted from cysts was multiplied by 10 (for the dilution) and fitted to the validated linear regression model. In order to establish the number of eggs per g soil, the number of eggs 
212 obtained from qPCR was divided by $400 \mathrm{~g}$ (dry weight of the soil sample from the field). For

213 standardizing the egg numbers between plots and fields, the data is presented as a

214 multiplication ratio of the population at the sampling time point divided by the initial number

215 of eggs in that experimental plot.

216

217 Hatching of field cysts

218 Cysts were recovered from soil samples collected at the final harvest and subjected to a

219 period of at least 3 months at $4{ }^{\circ} \mathrm{C}$. Hatching experiments were performed on a thermal

220 gradient table following the protocol of Kaczmarek et al. $(2014 \mathrm{a}, \mathrm{b})$ with a temperature range

221 from 5 to $19^{\circ} \mathrm{C}$.

222

223 Statistical analysis

224 Data was transformed and analysed using GENSTAT v. 17.1 and EXCEL v. 14.0.4760.1000 and

225 v. 16.0.4456.1003. Data (numbers of J2s, males, cysts and eggs) were analysed using

226 standard analyses of variance, linear and logarithmic regression as well as $t$-tests as detailed

227 in the text. The data were subjected to logarithmic transformations where specified. The

228 hatching data was analysed following the procedures in Kaczmarek et al. (2014a, b).

229

230 Results

231 Occurrence of juveniles, males, and cysts in the soil under two different temperature

232 regimes 
233 The first presence of juveniles in the soil was recorded at week 1 for both cvs Desirée and

234 Morag and in both the 14.3 and $17.3{ }^{\circ} \mathrm{C}$ temperature regimes, after 62 and $80 \mathrm{DD}$

235 respectively. This was followed by an increase in numbers and then a subsequent decrease

236 after week 2 (between 104 and 295 DD; Fig. 1a,b). Around 1219 DD (week 10 at $17.3{ }^{\circ} \mathrm{C}$ ),

237 increasing numbers of J2s were recorded with Desirée (Fig. 1b) until the end of the

238 experiment at week $16(1500 \mathrm{DD})$, with comparable numbers $(51 \pm 23 \mathrm{~J} 2$ per pot $)$ to those

239 observed during the first hatching peak. A small and later second hatch was also observed at

$24014.3^{\circ} \mathrm{C}$ at $1019 \mathrm{DD}$ (week 14; Fig. 1a) with Desirée. Juveniles recovered from the pots with

241 Morag, kept the same hatching trend as susceptible Desirée. The fewest numbers of juveniles

242 were observed for Morag at the cooler temperatures.

The first appearance of males in both temperature regimes was at week 5 (332 DD)

244 (Fig. 1c, d) with the highest numbers of males at $14.3{ }^{\circ} \mathrm{C}$ on Desirée at week 6 (436 DD)

245 whereas fewer were observed on Morag at $14.3^{\circ} \mathrm{C}$ at week 6 . At $17.3{ }^{\circ} \mathrm{C}$, males were

246 observed again at weeks 11, 13, 14, 15 and 16 with Desirée and at week 15 with Morag.

247 Males were not observed at $14.3{ }^{\circ} \mathrm{C}$ for either cultivar after week 11.

Mature (tanned) cysts were recovered from the soil from 9 weeks after inoculation onwards with Desirée at $14.3{ }^{\circ} \mathrm{C}(655 \mathrm{DD})$ and $17.3{ }^{\circ} \mathrm{C}(844 \mathrm{DD})$, with greater numbers at the higher temperature (Fig. 1e), peaking at 938 DD. Very few cysts were recovered with Morag at either temperature. There was a significant difference in the overall mean number of cysts recorded between the two temperature regimes $\left(14.3\right.$ and $\left.17.3{ }^{\circ} \mathrm{C}\right)$, the mean responses at different temperatures $(P=0.005)$ and for different cultivars $(P<0.001)$. There was also an interaction between temperature and cultivar $(P=0.007)$. 
258 The average soil temperatures at a depth of $20 \mathrm{~cm}$ in the potato drills over the growing seasons of 147 and 157 days were 14.1 and $15.5^{\circ} \mathrm{C}$ at the East Lothian and Harper Adams sites, respectively (Table 1). The fluctuations in the soil temperature during the growing season at the 2 sites is shown in Fig. 2. The temperatures at Harper Adams were higher than at East Lothian during three of the periods between soil samplings and were particularly high during the final harvest period (average 16.6 compared to $12.9^{\circ} \mathrm{C}$ ). Assuming a base temperature of $4{ }^{\circ} \mathrm{C}$ for G. pallida (Ebrahimi et al., 2014), there were 1511 and 1804 DD at the East Lothian site and Harper Adams sites respectively. A $t$-test confirmed significant differences in the soil temperature between the sites $(P<0.001)$.

qPCR validation

268 The primers and probes for the qPCR assay were designed to be specific to G. pallida or G. rostochiensis (Reid et al., 2010) and were based on rDNA ITS sequences of many PCN isolates from Europe. All samples were tested for both PCN species but no G. rostochiensis was detected. In order to use this assay to quantify G. pallida in the field trial soil samples, it was necessary to establish that the relationship between egg numbers and qPCR qty was

273 linear over a wide range of eggs numbers. The DNA yield (qPCR qty) from G. pallida cysts

274 was positively correlated with the number of eggs (Fig. 3) giving the linear regression model: $275 y=25.367 x$ with $R^{2}=0.7619$, where $y$ is the number of eggs of G. pallida and $x$ is DNA yield 276 multiplied by 10 for the dilution. Once established, the model was applied to field samples to 277 determine the egg number per sample. 
279 The initial population (Pi) of the East Lothian field site was an average of $11.7 \pm 1.6$ (without nematicide plots) and $20.6 \pm 2.2$ (with nematicide plots) eggs per g soil of G. pallida.

281 Changes in the G. pallida population over the growing season, without and with nematicide treatment for the four cultivars are presented in Figure $4 \mathrm{a}$ and $4 \mathrm{~b}$, respectively. In the plots without nematicide treatment, the multiplication ratio (population at sampling:initial population) generally decreased at 4,8 and 12 weeks, which is consistent with a decrease in the egg content of cysts due to hatching. At week 16, the multiplication ratio peaked. At week 20 (1511 DD) the final PfPi (final population/initial population) dropped significantly and ranged from $5.0 \pm 1.9$ for Cara to $2.4 \pm 0.4$ for Desirée. However, in the nematicide-treated plots, the multiplication remained at similar levels over the whole growing season. The overall trend for the effect of nematicides is shown in Figure 4c, where the multiplication ratio at each sampling time has been averaged over the four cultivars. The final average PfPi values were $3.6 \pm 0.6$ for the untreated and $0.7 \pm 0.1$ for nematicide-treated plots. The relative increase in the population was clear without nematicide treatment at weeks 16 and 20 in contrast to the nematicide treatment where the final population was lower than the initial population. To assess the influence of nematicide treatment and cultivar on multiplication (PfPi), an ANOVA was used. It revealed no significant differences in the reproduction ratio between the cultivars during the growing season; however, there nematicide treatment had a significant influence on population multiplication $(P<0.001)$ over the growing season.

The Pi of G. pallida in the Harper Adams field trials was estimated prior to planting in April. The average of $9.9 \pm 0.6$ (without nematicide) and $6.3 \pm 0.9$ (with nematicide) eggs per g soil was determined by qPCR. Samples were negative when tested for G. rostochiensis. Changes in the G. pallida population over the growing season in the plots without and with nematicide treatment for the four cultivars are presented in Figure 5a and 5b respectively. For samples 
without nematicide treatment, there were generally slight reductions in the population at 4 and 8 weeks after planting compared to preplant levels. At week 16 (1193 DD), the multiplication ratio increased to reach the PfPi at final harvest of $7.7 \pm 3.0$ for Cara and $2.5 \pm$ 0.5 for Estima. For the nematicide treatment, the levels remained similar for the first 12 weeks of the field experiment, but the multiplication ratio increased at the fourth sampling time and at the combined harvests at weeks 20 and 22. The final average PfPi values for the four cultivars were $5.2 \pm 1.0$ and $2.1 \pm 0.3$ for the untreated and treated plots, respectively (Fig. 5c). To assess the influence of nematicides and different cultivars on multiplication, an ANOVA was performed. There were no significant differences in the PfPi between the cultivars during the growing season; however, there was a significant influence of nematicide treatment on PfPi $(P<0.001)$ over the growing season.

\section{Relationship between initial population and reproductive factor (Pf/Pi)}

316 The Harper Adams site was lightly to moderately infested with G. pallida $(<20$ eggs per g 317 soil) and the site in East Lothian varied from lightly to heavily infested with a range of initial 318 population densities (5-50 eggs per g soil). The results indicated that in the untreated plots at 319 both sites, particularly at East Lothian, there was a trend towards a decreasing multiplication rate with increasing initial population (Fig. 6a). The multiplication rate was significantly higher in the plots with lower initial densities $(P<0.001)$. Also, the multiplication rate (PfPi) was reduced with the oxamyl treatment at the East Lothian site. At the Harper Adams trial, no trend was found between Pi and PfPi in the nematicide treated plots (Fig. 6b). Further statistical tests (ANOVA) on the multiplication rate revealed a significant effect of initial population on the PfPi $(P<0.001)$ and nematicide treatment $(P<0.001)$ at both sites but no difference in multiplication rate associated with different cultivars. 
328 At the first harvest (week 4; 281 DD) from the East Lothian site, J2 nematodes were most 329 frequently observed in the roots in both nematicide-treated and untreated samples, although 330 single J3 and J4 stages were also found (Fig. 7a, b). At week 8 (593 DD), mainly J3 stage 331 nematodes were observed, and much lower numbers of $\mathrm{J} 2$ and a few $\mathrm{J} 4$ stage nematodes.

332 There was an increase in the J2 nematodes at week 12 (895 DD), particularly in Desirée 333 roots.

At Harper Adams, J2 nematodes were observed in the roots of both Desirée and Cara at week 4 (278 DD; Fig. 8a, b). The highest number was recorded in Cara from the untreated plots. At week 8 (544 DD), both J3 and J4 stage nematodes were observed. J2 and J3 stages were observed in Cara samples from week 12 (856 DD) in non-nematicide-treated plots, while no nematodes were found in roots from the treated plots. Juvenile stage 2 nematodes were observed to increase in numbers in untreated plots at weeks 20 and 22 (end of experiment 1804 DD).

In the East Lothian root samples, the first occurrence of females and highest numbers were found on roots from Cara and Desirée 8 weeks (593 DD) after planting (Fig. 7c). Lower numbers of females were seen at week 12 (895 DD); however, at week 16 (1189 DD) no females were observed. Surprisingly the highest numbers of females were found with the nematicide treatment. At week 20 the roots had deteriorated, and it was not possible to examine them histologically. A few females were observed on the roots of Cara at week 4

347 (278 DD) at the Harper Adams site, but most were seen at 8 (544 DD) and 12 weeks (856

348 DD), with higher numbers observed without nematicide treatment (Fig. 8c). Females were 349 found at combined weeks 20 and 22 (1804 DD) on the roots of Cara and Desirée in the untreated samples, and a few were seen at week 22 (1804 DD) in roots from the nematicide treatment with both cultivars. 
$353 \quad$ Hatching test with field cysts

354 The cumulative proportion of eggs that hatched from the field cysts from the two sites was

355 calculated for each of the sets of cysts over the 56 days of the experiment (Fig. 9a) and fitted

356 to a linear regression model. Analysis of variance on the log-transformed curve parameters

357 suggested no evidence of differences in the means between the two sites (East Lothian and

358 Harper Adams) for maximum hatch rate or time to $50 \%$ hatch. There was a (marginally)

359 significantly higher total proportion hatching at East Lothian than Harper Adams $(P<0.001)$

360 (Fig. 9b). This is confirmed by ANOVA on the final counts (raw data), where the mean total

361 hatch at East Lothian was 975 and for Harper Adams $711(P=0.03)$, and ANOVA on the

362 total proportion hatching. There are also significant differences in the time to $50 \%$ hatch and

363 total hatch between temperatures but not for hatching rate.

365 Discussion

366 The main goal of these experiments was to investigate the relationship between the life cycle

367 of G. pallida and temperature and to assess the potential for the development of a second

368 generation of G. pallida in UK fields. The data has also been used for the development of a

369 dynamic stage-structured simulation model (Kettle \& Nutter, 2015) and for risk assessments

370 in relation to climate change (Skelsey et al., 2018). The growth cabinet experiments provide a

371 temporal framework for the rates of development in different temperature regimes and

372 indicate the relative abundance of the different life stages in different temperature regimes.

373 The pot experiment was performed to allow comparison between the life cycle of G. pallida

374 in a controlled environment and in more variable field conditions. There was no effect of the 
375 potato cultivar on the number of juveniles recovered from the soil in the initial hatching peak.

376 This is in agreement with Turner (1990) who found no significant difference in hatching of

377 G. pallida with various S. vernei hybrids. In previous in vitro hatching experiments

378 conducted in petri dishes using potato root diffusate to induce hatching(Kaczmarek et al.,

379 2014a, b)), newly hatched juveniles of G. pallida were first observed at day 9; however,

380 more hatched by day 12 at $13-25{ }^{\circ} \mathrm{C}$. The results obtained with the growth cabinet experiments using potato plants in soil, showed a similar delay before hatching occurred observed by the presence of juvenile nematodes recovered from the soil, and hatching continued for several weeks. Adult males were recovered from the soil at $333 \mathrm{DD}$ and the maximum number of males was recorded about 430 DD in both temperature regimes; however, more males were recorded for the growth cabinet with the lower temperature. Further investigation is needed to determine if there is an effect of temperature on sex determination (Jones et al., 2017; Skelsey et al., 2018).

In the present investigation, new cysts were observed in the $17.3^{\circ} \mathrm{C}$ regime from nine weeks after inoculation (844 DD), followed by the start of a second increase in the number of juveniles in the soil at week 10 (938 DD), indicating that fewer than 10 weeks were required for one generation to be completed and for another to start in these conditions for this population of G. pallida. The juveniles observed at 10 weeks (938 DD) and later are consistent with the hatching of a second generation originated from new, first generation females; this implies that diapause was not obligatory for all juveniles from the first generation, although the amount and timing of the second hatch was affected by the temperature regime. Hatching with PCN is not tightly synchronized, resulting in the

397 occurrence and overlapping of different developmental stages over several weeks.

398 Nevertheless, based on these results, if the second generation develops at the same rate as the first generation at this temperature, then second generation cysts would be expected at week 
400

401

402

403

404

405

406

407

408

409

410

411

412

413

414

415

416

417

418

419

420

421

422

423

18 (about 8 weeks after the second appearance of juveniles in the soil). Because it was not possible to sustain the plants for longer than 16 weeks in the growth cabinets despite regular fertilization and watering, it was not possible to determine whether a second increase in cysts would occur.

In both temperature regimes, lower numbers of cysts were observed with partially resistant Morag compared to Desirée, consistent with previous reports (Phillips et al., 1980; Phillips \& Trudgill, 1998). However, there was a much greater response to temperature with susceptible Desirée. This indicates that partially resistant cultivars may be more effective than susceptible cultivars in controlling population multiplication over a wider temperature range. Significantly lower egg numbers per cyst were found with Morag than with Desirée, but numbers did not differ significantly between the two temperature regimes. This contrasts with other reports (Bendezu et al., 1998; Da Cuhna et al., 2012) where resistance from $S$. vernei did not significantly reduce the number of cysts or egg content in more virulent populations of G. pallida from Portugal and Bolivia, although with this source of resistance, less virulent populations of G. pallida had significant differences in their multiplication.

The experiments in temperature-controlled environments described here used a PCN population from the JHI collection that has been multiplied in glasshouse conditions and may have become adapted to these conditions. Other work has noted differences between populations of G. pallida in their temperature responses (Foot, 1978) and a population of $G$. rostochiensis was selected that matured more quickly at low temperatures following several years of early cropping in Ayrshire, UK (Ellenby \& Smith, 1975). However, the hatching tests over a range of temperatures performed in the present study with G. pallida cysts isolated from two field sites confirmed the responses obtained previously with glasshouse cultured G. pallida (Kaczmarek et al., 2014 a, b). 

monitor the densities of eggs per g soil in soil samples taken at the two field sites. This is the

426

427

first time this assay has been used to monitor PCN population dynamics in the field over a growing season and demonstrates the value of this high-throughput assay for assessing the impact of different PCN control programmes. The relationship between egg numbers and DNA yield was validated using G. pallida cysts recovered and quantified from soil using the same extraction procedure used for the field samples.

The mean soil temperature between planting and first harvest was $13.9^{\circ} \mathrm{C}$ at the Scottish site compared to $14.4{ }^{\circ} \mathrm{C}$ at the English site, below the optimal temperatures that have been observed for hatching (Kaczmarek et al., 2014a, b). At harvest 1 at East Lothian but not in the Harper Adams root samples, J3 stage nematodes were observed and a few female nematodes were observed on the roots from Harper Adams. The mean soil temperatures in sampling periods 1 and 2 were lower at Harper Adams than the East Lothian site; however, they increased to 15.5 and $16.6^{\circ} \mathrm{C}$ in sampling periods 4 and 5 giving 1193 and $1804 \mathrm{DD}$ respectively, whereas they dropped to 14.4 and $14.1^{\circ} \mathrm{C}$ at the East Lothian site with 1189 and 1511 DD respectively. Females were observed on the roots from week 4 onwards at Harper Adams with relatively warmer soil temperatures $\left(14.8-15.5^{\circ} \mathrm{C}\right)$ as found by Jones et al. (2017). It is probable that a second generation developed, as both juveniles and females were observed at harvests 5 (week 20) and 6 (22 weeks), although the possibility that the late females observed in the field samples may have resulted from a delayed initial hatch cannot be discounted. However, two generations of G. pallida in a growing season has been reported with high numbers of cysts in soil temperatures from 18 to $22{ }^{\circ} \mathrm{C}$ in Avezzano, Italy (Greco et al., 1988) and in the present study, a second hatch was observed at 1219 DD in the controlled environment cabinet at $17.3{ }^{\circ} \mathrm{C}$. Ebrahimi et al. (2014) estimated the degree day requirement for G. pallida of $450 \mathrm{DD}$ with a $4{ }^{\circ} \mathrm{C}$ base temperature. There were $1511 \mathrm{DD}$ 
and 1842 DD at the East Lothian and Harper Adams sites respectively, both sufficient for two generations to be completed.

The general trend of increasing temperatures associated with climate change combined with the warmer conditions that occur in some regions in the UK are making it more likely that conditions that support two generations of $G$. pallida within a growing season will occur. A recent report showed that both species of PCN are developing more quickly in Belgium than in the past (Ebrahimi et al., 2014) and this has resulted in a change of advice for the time of harvesting early cultivars to prevent PCN from completing its life cycle. In 2014 in Lincolnshire, average soil temperatures at a depth of $20 \mathrm{~cm}$ of $15.3{ }^{\circ} \mathrm{C}$ were recorded and many females were observed developing on roots of susceptible untreated potatoes 21 weeks after planting (A. Barker, Barworth Agriculture LTD, Sleaford, personal communication). It is not known whether these late developing females matured into cysts or whether they contained viable eggs that could contribute to a higher final PfPi; this requires further investigation. In some conditions, a partial second generation, where a substantial number of nematodes hatch but are not able to complete the second generation of mature cysts, could lead to lower final PfPi values and a trap crop effect. An effect of temperature on in-egg mortality and spontaneous hatching, affecting population dynamics, has also been reported (Devine et al., 1999).

Nematicides can affect PCN during different developmental stages, mainly affecting juvenile nematodes before they reach the potato plants. Fosthiazate temporarily inhibited hatching of G. pallida in an in vitro test and suppressed hatching in soil (Woods et al., 1999). The results of the nematicide trial in the current investigation are consistent with other studies that have reported that carbamate nematicides do not permanently stop hatching in field conditions (Evans \& Wright, 1982; Woods et al., 1999); nevertheless, they do delay the 
473 process significantly. Although the decline in egg numbers was less in nematicide treated

474 plots than in untreated plots, this was probably due to less hatching with the nematicide

475 treatment. Generally, fewer J2 stage nematodes were observed in the roots of Cara and

476 Desirée with nematicide treatment, consistent with a report by Minnis et al. (2004) and fewer

477 females were observed at week 8 with the nematicide treatments at both sites. At the East

478 Lothian site there was significantly lower multiplication with the oxamyl treatment $(P<$

479 0.001). This is in contrast to the results of Minnis et al. (2004) that showed no significant

480 difference in PCN multiplication between fields treated or untreated with oxamyl. However,

481 as mentioned previously, in the current investigation, the density of the initial population in

482 untreated plots ranged from 5 to 20 eggs per g soil while the plots for nematicide treatment

483 had $>20$ eggs per $\mathrm{g}$ of soil; therefore, it is difficult to differentiate between a reduction in the

484 multiplication rate due to the nematicide treatment or due to the higher initial number of eggs

485 in the soil.

Understanding how climate change may drive the life cycle and population dynamics

487 of PCN is important because of the role of these plant pests in potato yield reduction; in

488 addition, the management of this pest is of increasing concern due to the withdrawal of

489 nematicides from use. The results indicate that the development of multiple generations of

490 PCN within one growing season in the UK is more likely with increasing soil temperatures

491 associated with climate change and this could have significant effects on management of

492 population multiplication and prevention of the spread of PCN (Jones et al., 2017; Skelsey et

493 al., 2018).

494

495 Acknowledgements 
The authors gratefully acknowledge the assistance of their James Hutton Institute colleagues

497 Finlay Dale, Ralph Wilson, Alison Paterson and Anne Holt. They also gratefully

498 acknowledge the assistance of staff at SASA who helped with cyst extraction and Alex Reid who provided guidance on the qPCR assay, as well as students and staff at Harper Adams

500 University (Jennifer Heath, Nelson Opoku and Matthew Rodenhurst) who helped with the 501 field trials. The authors wish to pay tribute to the late Dr Pat Haydock who was instrumental in the early stages of this project. The provision and management of the East Lothian trial site by Allan Stevenson and Geert Knottenbelt is also acknowledged. Funding for this work was

504 received from the Agriculture and Horticulture Development Board, and The James Hutton 505 Institute received funding from the Rural and Environment Science and Analytical Services 506 (RESAS) Division of the Scottish Government.

\section{References}

Bendezu IF, Russell MD, Evans K, 1998. Virulence of populations of potato cyst nematodes (Globodera spp.) from Europe and Bolivia towards differential potato clones frequently used for pathotype classification. Nematologica 44, 667-81.

Bridge J, Page S, Jordan S, 1981. An improved method for staining nematodes in roots. Report of the Rothamsted Experiment Station 1981, 171.

Brodie BB, Blow RE, Brace NL, King JH, 1976. A new technique for handling cysts of Heterodera rostochiensis during routine laboratory procedures. Plant Disease Reporter 60, 936-8.

Devine KJ, Dunne C, O’Gara F, Jones PW, 1999. The influence of in-egg mortality and spontaneous hatching on the decline of Globodera rostochiensis during crop rotation in 
520

521

522

523

524

525

526

527

528

529

530

531

532

533

534

535

536

537

538

Ebrahimi N, Viaene N, Demeulemeester K, Moens M, 2014. Observations on the life cycle of potato cyst nematodes, Globodera rostochiensis and G. pallida, on early potato. Nematology 16, 937-52.

Ellenby C, Smith L, 1975. Temperature adaptation in the potato cyst nematode, Heterodera rostochiensis. Nematologica 21, 114-5.

Elston DA, Phillips MS, Trudgill DL, 1991. The relationship between initial population density of potato cyst nematode Globodera pallida and the yield of partially resistant potatoes. Revue de Nématologie 14, 213-20.

EPPO, 2019. EPPO Global Database. (https://gd.eppo.int/) (12/2/19) htm].

Evans K, 1993. New approaches for potato cyst nematode management. Nematropica 23, $221-31$.

Evans SG, Wright DJ, 1982. Effects of the nematicide oxamyl on life cycle stages of Globodera rostochiensis. Annals of Applied Biology 100, 511-9.

Foot MA, 1978. Temperature responses of three potato-cyst nematode populations from New Zealand. Nematologica 24, 412-7.

Greco N, Di Vito M, Brandonisio A, Giordano I, de Marinis G, 1982. The effect of Globodera pallida and G. rostochiensis on potato yield. Nematologica 28, 379-86.

Greco N, Inserra R, Brandonisio A, Tirro A, de Marinis G, 1988. Life-cycle of Globodera rostochiensis on potato in Italy. Nematologia Mediterranea 16, 69-73. 
541 Hooper DJ, 1970. Handling, fixing, staining and mounting nematodes. Technical Bulletin. Ministry of Agriculture, Fisheries and Food, 5th edn. 2, 39-54.

543 Jones FGW, 1983. Weather and plant parasitic nematodes. EPPO Bulletin 13, 103-10.

544 Jones LM, Koehler A-K, Trnka, M et al., 2017. Climate change is predicted to alter the

545

546

547

548

549

550

551

552

553

554

555

556

557

558

559

560 current pest status of Globodera pallida and G. rostochiensis in the United Kingdom. Global Change Biology 23, 4497-507.

Kaczmarek A, MacKenzie K, Kettle H, Blok VC, 2014a. Increasing soil temperatures will likely benefit potato cyst nematodes. In: Crop Protection in Northern Britain 2014. Dundee, UK: Association for Crop Protection in Northern Britain, 265-70.

Kaczmarek A, McKenzie K, Kettle H, Blok VC, 2014b. The influence of temperature on the plant parasitic nematodes Globodera rostochiensis and G. pallida. Assessing the impact of soil temperature. Phytopathologia Mediterranea 53, 396-405.

Kettle H, Nutter D, 2015. STAGEPOP: Modelling stage-structured populations in R. Methods in Ecology and Evolution 6, 1484-90.

Minnis ST, Haydock PPJ, Evans K, 2004. Control of potato cyst nematodes and economic benefits of application of 1,3-dichloropropene and granular nematicides. Annals of Applied Biology 145, 145-56.

Munir A, Phillips MS, Trudgill DL, Shahina F, Kazmi AR, Firoza K, 2009. Effect of exposure to high temperatures on the hatching and reproduction of Globodera rostochiensis. Pakistan Journal of Nematology 27, 203-11. 
Phillips MS, Trudgill DL, 1998. Variation of virulence, in terms of quantitative reproduction of Globodera pallida populations, from Europe and South America, in relation to resistance from Solanum vernei and S. tuberosum ssp. andigena CPC 2802. Nematologica 44, 409-23.

Reid A, Kenyon DM, Evans FF et al., 2010. Development of a high-throughput method for the detection and species determination of PCN. Aspects of Applied Biology 103, 13-6.

Seinhorst JW, 1982. The relationship in field experiments between population density of Globodera rostochiensis before planting potatoes and yield of potato tubers. Nematologica 28, 277-84.

Skelsey P, Kettle H, MacKenzie K, Blok V, 2018. Potential impacts of climate change on the threat of potato cyst nematode species in Great Britain. Plant Pathology 67, 909-19.

Stanton JM, Sartori M, 1990. Hatching and reproduction of the potato cyst nematode, Globodera rostochiensis, from potato fields in Western Australia as influenced by soil temperature. Nematologica 36, 457-64.

Trudgill DL, 1986. Yield losses caused by potato cyst nematodes - a review of the current position in Britain and prospects for improvements. Annals of Applied Biology 108, $181-98$.

Trudgill DL, Cotes LM, 1983. Tolerance of potato to potato cyst nematodes (Globodera rostochiensis and Globodera pallida) in relation to the growth and efficiency of the root-system. Annals of Applied Biology 102, 385-97.

581 Trudgill DL, Phillips MS, Elliott MJ, 2014. Dynamics and management of the white potato cyst nematode Globodera pallida in commercial potato crops. Annals of Applied 
584

585

586

587

588

589

590

591

592

593

594

595

596

597

598

599

600

601 given in brackets. Two pots were used for each temperature and cultivar combination, and

602 nematodes were extracted with a Baermann funnel. The bars indicate the standard error of 603

Turner SJ, 1990. The identification and fitness of virulent potato cyst-nematode populations (Globodera pallida) selected on resistant Solanum vernei hybrids for up to eleven generations. Annals of Applied Biology 117, 385-97.

Twining S, Clarke J, Cook S et al., 2009. Pesticide availability for potatoes following revision of Directive 91/414/EEC: impact assessments and identification of research priorities. Project Report 2009/2. Oxford, UK: Potato Council.

Viglierchio DR, Schmitt RV, 1983. On the methodology of nematode extraction from field samples: Baermann funnel modifications Journal of Nematology 15, 438-44.

Woods SR, Haydock PPJ, Edmunds C, 1999. Mode of action of fosthiazate used for the control of the potato cyst nematode Globodera pallida. Annals of Applied Biology 135, $409-15$.

\section{Figure legends}

Figure 1 Comparison of number of Globodera pallida juveniles at $14.3{ }^{\circ} \mathrm{C}$ (a) and $17.3{ }^{\circ} \mathrm{C}$ (b), males at $14.3{ }^{\circ} \mathrm{C}(\mathrm{c})$ and $17.3{ }^{\circ} \mathrm{C}(\mathrm{d})$, and cysts at 14.3 and $17.3{ }^{\circ} \mathrm{C}(\mathrm{e})$ recovered from the soil over 16 weeks in the growth cabinet experiment with susceptible cv. Desirée and partially resistant cv. Morag. The number of degree days (DD) at each sampling time are means. 
604 Figure 2 Soil temperatures at $20 \mathrm{~cm}$ in potato drills over the growing season in Harper 605 Adams (4 April 2011 to 6 September 2011, 155 days) and East Lothian (3 May 2011 to 28

606 September 2011, 147 days). Soil temperatures were taken every $3 \mathrm{~h}$ in two adjacent drills and 607 the averages are shown.

608 Figure 3 Linear regression between egg numbers of Globodera pallida and quantity of 609 copies determined by qPCR. The qPCR results for 11 samples were averaged across three 610 qPCR replicates.

611 Figure 4 Changes in the multiplication ratio (population at sampling time/initial population)

612 of Globodera pallida over the growing season (a) without nematicide treatment, (b) with 613 nematicide treatment, and (c) average multiplication ratio for the four cultivars (Cara,

614 Desirée, Estima and Maris Piper) with and without nematicide treatments at the East Lothian 615 site. Soil samples were taken at planting and at 4-weekly intervals (degree days (DD) are shown in brackets after each sampling time) and the eggs per $g$ soil determined from isolated

617 cysts using qPCR. The bars are standard errors of the means for each cultivar.

618 Figure 5 Changes in the multiplication ratio (population at sampling time/initial population)

619 of Globodera pallida over the growing season (a) without nematicide treatment, (b) with 620 nematicide treatment and (c) average final population/initial population (Pf/Pi) for the four 621 cultivars (Cara, Desirée, Estima and Maris Piper) with and without nematicide treatments at the Harper Adams site. Soil samples were taken at planting and at 4-weekly intervals (degree days (DD) are shown in brackets after each sampling time) and the eggs per g soil determined

624 from isolated cysts using qPCR. The bars are standard errors of the means for each cultivar.

625 Figure 6 Reproductive factor (ratio of final population/initial population (PfPi)) versus initial population (Pi) of Globodera pallida at (a) East Lothian and (b) Harper Adams sites. 
627 Figure 7 The numbers of J2, J3, J4 and females of Globodera pallida observed inside $100 \mathrm{~g}$ 628 of acid fuchsin-stained roots of cultivars Cara and Desirée from the 2011 field trials at East 629 Lothian. (a) Non nematicide-treated plots, (b) nematicide-treated plots, and (c) females from 630 both treatments. Root samples were examined at harvests 1, 2, 3 and 4 (weeks 4, 8, 12 and 631 16, respectively, which correspond to 281, 593, 895 and 1189 degree days (DD)). The bars 632 indicate the standard error of the mean.

633 Figure 8 The numbers of J2, J3, J4 and females of Globodera pallida observed inside $100 \mathrm{~g}$ 634 of acid fuchsin-stained roots of cultivars Cara and Desirée from 2011 field trials at Harper 635 Adams; (a) non-nematicide-treated plots, (b) nematicide-treated plots, (c) females from both 636 treatments. Root samples were examined at harvests 1, 2, 3, 4, 5 and 6 (weeks 4, 8, 12, 16, 20 637 and 22, respectively, which correspond to $278,544,856,1193$ and 1804 degree days). The 638 bars indicate the standard error of the mean.

639 Figure 9 Proportion of hatched eggs of Globodera pallida from the field populations in 640 potato root diffusate, during 55 days incubation at different temperatures; (a) cumulative and 641 (b) percentage of total hatch. Bars indicate standard errors of means. 